\title{
Prediction of Toxic Emissions from Chemical Fire and Explosion
}

\author{
N. ALBERT MOUSSA and VIJAY V. DEVARAKONDA \\ BlazeTech Corp. \\ 29B Montvale Ave., Woburn, MA 02421, USA
}

\begin{abstract}
The prediction of toxic emissions from industrial and warehouse fires and explosions involving reactive chemicals has eluded the hazard analysis community for quite some time. To address this issue, we developed a model called ADORA for the time evolution of toxic emissions and their dispersion in the atmosphere. At each time step, the conservation of mass, energy and momentum are solved while invoking thermochemical equilibrium or a constrained version thereof to determine the species composition in the cloud. During the initial stages of cloud evolution, the temperatures are usually high and thermochemical equilibrium applies. As the cloud cools down later due to air entrainment, the composition is governed by reaction kinetics. We use a computationally efficient approach called "constrained equilibrium" which is essentially an approximate way of accounting for temperature dependent reaction kinetics. In this approach, as air and moisture are entrained into the plume, the species concentrations are updated until the temperature decreases sufficiently to "freeze out" the toxic species of concern. The freeze-out temperatures for the toxic species of greatest concern are determined by examining the temperature dependent reaction kinetic rates. The temperatures below which the kinetics are too slow relative to cloud dynamics are selected as freeze out temperatures. This approach allows us to calculate as a function of time the cloud combustion rate, temperature, species composition, size, rise and travel distance downwind from the release location. Sample calculations for the detonation of explosives and for fires involving several reactive chemicals are given. For the former, the model predictions of major species such as carbon dioxide, carbon monoxide, nitrogen oxides and total non-methane hydrocarbons agree well with the limited available data. The model predicts the time dependent consumption of reactants and formation of reaction intermediates as well as stable end products. The concentration contours for toxic species such as hydrogen fluoride are presented and the trends discussed. The predictions of our model can be used to improve preparedness and emergency response planning in order to minimize the consequences of accidents involving reactive and energetic materials.
\end{abstract}

KEYWORDS: chemical warehouse fires, energetic materials, reactive dispersion, toxic hazards, chemical plant fires, explosions

\section{INTRODUCTION}

The importance of toxic emission from fires was recognized in the TOXFIRE project performed collaboratively between partners from United Kingdom, Sweden, Finland and Denmark in 1994-1996 [1]. They recognized that chemical warehouse fires are economically impossible to study at the full scale. Accordingly, they characterized the combustion products from burning of the same materials at the micro, small, medium, and a room scale. These materials were polypropene, nylon 66, tetramethylthiuram monosulfide (TMTM), 4-chloro-3-nitrobenzoic acid (CNBA), and chlorobenzene (CB), selected for their potential toxic emission. They measured the mass of combustion products per mass of material consumed, which is called yield or emission factor. They found that this yield depended on the amount of material burned and other factors such as ventilation and hence could not be simply scaled.

For common hydrocarbon fuels, NIST performed an extensive effort to measure emission factors for CO, $\mathrm{CO}_{2}$, NOx, $\mathrm{SO}_{2}$, Volatile Organic Compounds (VOCs), Poly Aromatic Hydrocarbons (PAHs) and smoke, for the burning of various types of crude oil, diesel and emulsified coal [2 -6]. Such emission factors for various pollutants $\left(E F_{i}\right)$ were used by Argyropoulos and co-workers [7 and 8] in the analysis of the Buncefield Oil Depot Fire of 2005. They used a CFD model to calculate the fuel burning mass flux $\left(\dot{m}^{\prime \prime}\right)$ and area $\left(A_{f}\right)$; then gave the source terms $S_{i}$ for each pollutants as:

$S_{i}=\dot{m}^{\prime \prime} \times A_{f} \times E F_{i}$ 
They recognized several drawbacks in their approach, namely variations in the emission factors due to differences in scale, fuel chemistry, fuel configuration, and atmospheric conditions between the NIST data and the Oil Depot conditions. Still, for lack of anything better, Mohan et al used these source terms in the NAME atmospheric model to forecast toxic hazards downwind under different meteorological conditions for the Buncefield Oil Depot Fire [9].

When measurements of emission factors are not available or possible, one resorts to modeling. The classical approach is to couple a Computational Fluid Dynamic (CFD) code with chemical reaction kinetics. In order to reduce the stiffness of the equations as well as the computational effort involved in such calculations, detailed chemical reaction schemes consisting of a large number of elementary reactions are usually simplified to a reduced set with fewer reactions. This approach has been successful with simple fuels (such as hydrogen and methane) under well controlled combustion conditions (such as engines) for which there were lot of validation data.

Applying this CFD with chemical reactions approach to fires which involve more complex fuels and conditions is intractable mainly because the kinetics are unknown. Altarawneh and Dlugogorski presented a recent review of the fundamental mechanisms of pollutant formation from biomass, plastics and polyfluorocarbons fires [10]. They recognized that the overall emission is driven by chemical reactions, physical processes such as devolatilisation, transport phenomena (momentum, heat and mass transfer) and aerodynamics. Focusing uniquely on chemical reactions, they summarized qualitatively the mechanisms pertinent to the formation of prominent pollutants, such as $\mathrm{CO}, \mathrm{NOx}, \mathrm{HCN}, \mathrm{HCl}$, soot, and trace species such as polychlorinated dibenzo dioxins and furans. However, they gave no kinetic parameters that can be used in predictions. In fact they stated "it is a colossal task to identify the precise source and the governing mechanism of emissions" [10].

For the limited cases when the kinetics parameters are known, one has to still overcome the equation stiffness issue. One approach is illustrated by the recent work of Tuovinen et al [11 and 12] to model HCN formation from the burning of Nylon in a room fire. They represented Nylon by a mixture of ethylene and methylene. Using 1000s of elementary reactions, they developed a chemical library consisting of precalculated instantaneous state relationships for a number of flamelet conditions predicted by the CFD. The flamelets conditions varied in equivalence ratios, temperatures, vitiation levels, turbulent intensities, radiation losses, and strain rates up to extinction. In each calculation step in the CFD, the pre-calculated chemical state relationships were called from the library based on conditions within the fluid, eliminating the need to calculate reaction rates on the spot.

Given the importance of HCN formation which is common in building fires, the chemical library approach is reasonable for room fires. In a warehouse involving a large number of chemicals which differ significantly in atomic composition, flammability and fire suppression requirements, the potential number of combinations of elementary reactions and fire conditions become unwieldy - making this approach intractable.

Of particular concern to the US Chemical Safety Board (CSB) are reactive chemicals with the potential of undergoing a violent, uncontrolled reaction when processed or handled improperly [13]. The chemical reactions can release large quantities of heat, energy and gases, causing fires, explosions and toxic emissions. A 2002 investigation by the CSB found 167 serious reactive incidents in the U.S. over the previous two decades. These incidents caused 108 fatalities as well as extensive property and environmental damage [13]. For example, halogenated compounds stored in bulk can be reactive and produce large quantities of toxic chemicals such as hydrogen chloride, hydrogen fluoride, chlorine and fluorine. Their ignition can lead to numerous separate events with distinct time scales and hazard footprints that can interact with each other. For example, as fire fighters approach to fight the primary fire, a secondary explosion or fireball can emerge exposing them to blast, heat and/or toxic gases. Also, the resulting toxic footprint could be one or two orders of magnitude larger than the thermal or blast hazard distances putting the public at risk.

In this paper we present a novel approach to simplify reaction kinetics while retaining their coupling to fluid dynamic processes. The approach is particularly suitable for explosions and fires involving reactive chemicals when the cloud is very hot initially. We have implemented this approach in a model called Atmospheric Dispersion Of Reactive Agents (ADORA). The model tracks the evolution of toxic products form an initial fire or explosion accounting for air entrainment, afterburn reactions, cloud rise, buoyant flow, atmospheric 
dispersion and deposition of solids (if any) downwind [14 and 15]. In this paper, we focus mainly on the novel treatment of chemical transformations in the cloud to predict the toxic species.

\section{MODEL DESCRIPTION}

ADORA calculates the composition and dispersion of the cloud resulting from reactive chemicals released into the atmosphere. It only needs reactants composition and local release and environmental conditions as inputs for these calculations. Assuming that the cloud shape is known (a spherical puff for an explosion as shown in Fig. 1 (a) and a plume for a fire as shown in Fig. 1 (b)), it solves the conservation of mass, momentum, energy and species equations in an integral formulation. The coupling between the cloud dynamics and chemical/thermodynamic transformations is accounted for in the model. The formulation of cloud dynamics (i.e., the fluid mechanics aspect of the overall solution) has been published in the past [14]. This paper focuses on the novel chemical formulation to predict toxic emission from fires and explosions.

Because of the similarities between explosions and fires, we discuss only the former below for brevity. The overall cloud evolution occurs in four stages. The first stage is the initial detonation that occurs over very short time scales ranging from microseconds to milliseconds. A large amount of energy is released very rapidly during this stage making the cloud very hot and buoyant. At these temperatures, the reaction rates are very fast and can be considered instantaneous. Classical thermochemical equilibrium is applied to determine the pool of species formed. It is in this initial period, before dilution has its cooling effect, where most of the dominant species are formed. If the fire involves halogenated compounds, most of the $\mathrm{HBr}$, $\mathrm{HCl}$ and $\mathrm{HF}$ are formed during this stage.

The second stage is afterburn reactions that start at the end of detonation and continue for a second or so. This is the most important step in terms of toxic chemical formation. During this step, the cloud rises up rapidly while traveling downstream with the ambient wind. It entrains ambient air at a rate that depends on cloud velocity. Depending on turbulence, a portion of this entrained air mixes with the detonation products leading to afterburn reactions that release additional heat. The cloud also loses heat due to a combination of cooling induced by entrained air, radiation and convection from the cloud as well as the work done by the cloud on the surroundings as it expands and rises up. The net effect of these various processes causes the cloud to cool down with time. If the fire involves halogens, species such as $\mathrm{Br}_{2}$ and $\mathrm{Cl}_{2}$ may become favored thermodynamically as the cloud cools down due to a shifting hydrogen balance in the product composition, while test data indicate a product distribution that more closely matches the initial, high temperature equilibrium that yields products such as $\mathrm{HF}$ and $\mathrm{HCl}$. Assuming that all the species formed during the initial higher temperature step remain frozen throughout the remaining dilution and cooling phases of puff evolution is a possible approach. However, the results from such over simplification are almost as meaningless as for the equally simplistic general equilibrium approach. We developed a compromise with a little insight into some kinetic issues. Our approach consists of freezing select species at specific temperatures dictated by the governing kinetics. For example, the $\mathrm{H}-\mathrm{X}$ bonds are known to be very strong with a high activation energy. If consumption of such chemicals were shut off early in the cloud formation process, the chemical composition results should more accurately represent reality. In its most generic form, the process consists of first identifying those species which may be lost prematurely to chemical reactions while actual kinetic activation energies are far too high for the equilibration mechanism to take place. HX would be used in the above example, but typically any relatively thermodynamically stable species (oxides such as $\mathrm{CO}$ and $\mathrm{NO}$ and halides such as $\mathrm{HF}$ and $\mathrm{HCl}$ ) are good freezing candidates. Note that the choice is only of major concern when there are more than one thermodynamically stable product species to partition into. After identifying candidate species, a reasonable temperature range such as $1000-2000 \mathrm{~K}$ where freezing may be appropriate are chosen. Another general rule to follow is that compounds with higher activation energies to destruction/decomposition (energy of the breaking bond can be used as an approximation) should possess proportionately higher freezing temperatures. In the absolute worst case where no data are available, and one wishes to be conservative about a particular species, that species should be frozen as early as possible in the explosion (at the highest temperature attained). Note that the chemical will still be allowed to accumulate, so this estimate for freezing temperature will produce the highest concentration attainable by the ADORA prediction. Preferably, however, a more moderate freezing temperature should be chosen to accurately model the chemistry. A parametric study within the reasonable temperature ranges is used to pinpoint accurate freezing temperatures to use given any data provided. Since it is based on comparison with actual data of interest, this method is accurate, reliable and 
more useful than the rather more complex, time-consuming and expensive process of estimating based on kinetic theory. If more than one species is to be frozen, their relative freezing temperatures should follow the trend in activation energy to destruction mentioned above. A good chemical judgment is all that is needed to make the freezing point decisions and to verify their consistency.

Once freezing temperatures are picked, the choice is used for all scenarios related to the chemical conditions of interest. New species or atoms, new data and/or drastically different release conditions would be required before a change is needed in the freezing temperature decisions. ADORA implements the freezing temperature concept in the following way. At any given time-step in the puff rise, if the temperature drops below the user-defined freezing threshold, the mass of the relevant species is taken out of consideration in future calls to the equilibrium calculation subroutine. While this original mass is not allowed to disappear, new mass for that species is allowed to accumulate in the usual way, again being taken out of consideration at each time step. This is done because we are only trying to prevent mass from disappearing due to kinetic limitations. Nothing is done to prevent the thermodynamically stable products from forming through energetic processes. While there is an acknowledged slight bias to form artificially excess stable product by this computation, one is reminded that this is the point of the freezing algorithm. Furthermore, most of the bias is removed if one pursues the recommended parametric study using real data. We validated this approach by comparing model predictions with data collected by the DoD from several military tests involving metals, halogens, and explosives.

The third stage is cloud rise that occurs because the contents are still hot and buoyant at the end of chemical reactions. It continues to rise, entrain air and disperse as shown in Fig. 1. This continues for several seconds until the cloud density becomes equal to the ambient air density. The fourth stage is passive dispersion that starts at the end of buoyant cloud rise. In this stage, the cloud stops to rise and undergoes dilution governed by atmospheric turbulence. The concentrations of various species in the cloud then decrease as the cloud disperses in the atmosphere.

We implemented the above modular approach in ADORA to calculate the cloud evolution all the way from the release to the end of atmospheric dispersion. This simplified formulation enables coupling the chemistry with the cloud dynamics in a computationally efficient manner without the need for detailed approaches such as CFD where coupling between processes that differ significantly in length and time scales is difficult. Using the amounts of various reactants and the environmental conditions as inputs, the model calculates the products of explosion or chemical fires, the air entrainment into the cloud and afterburn reactions, the heat release rates, the resulting cloud (plume or puff) dynamics and atmospheric dispersion. Thus, the cloud temperature, size, rise and trajectory, and the intermediate and final species composition and travel distance downwind from the release point are accurately calculated.

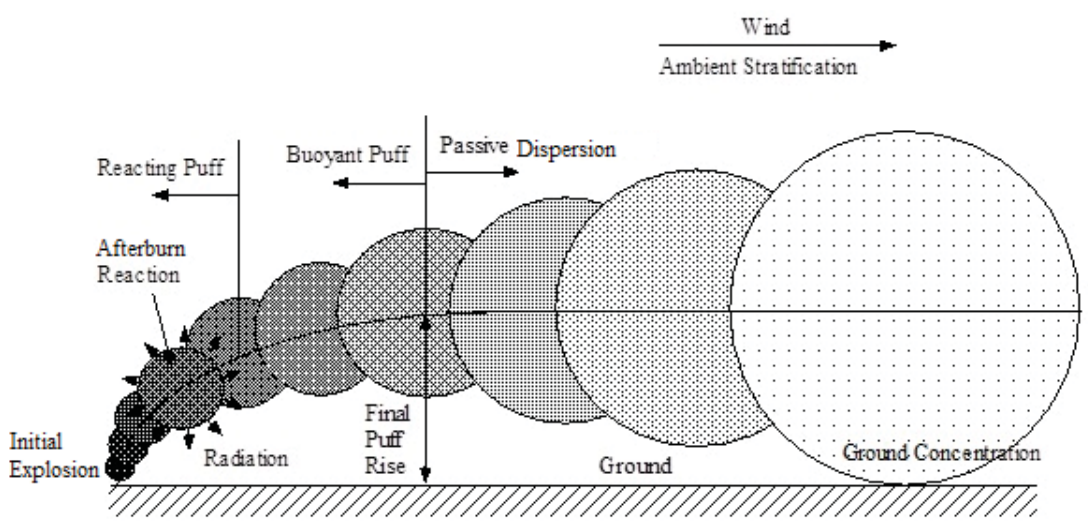

(a) 


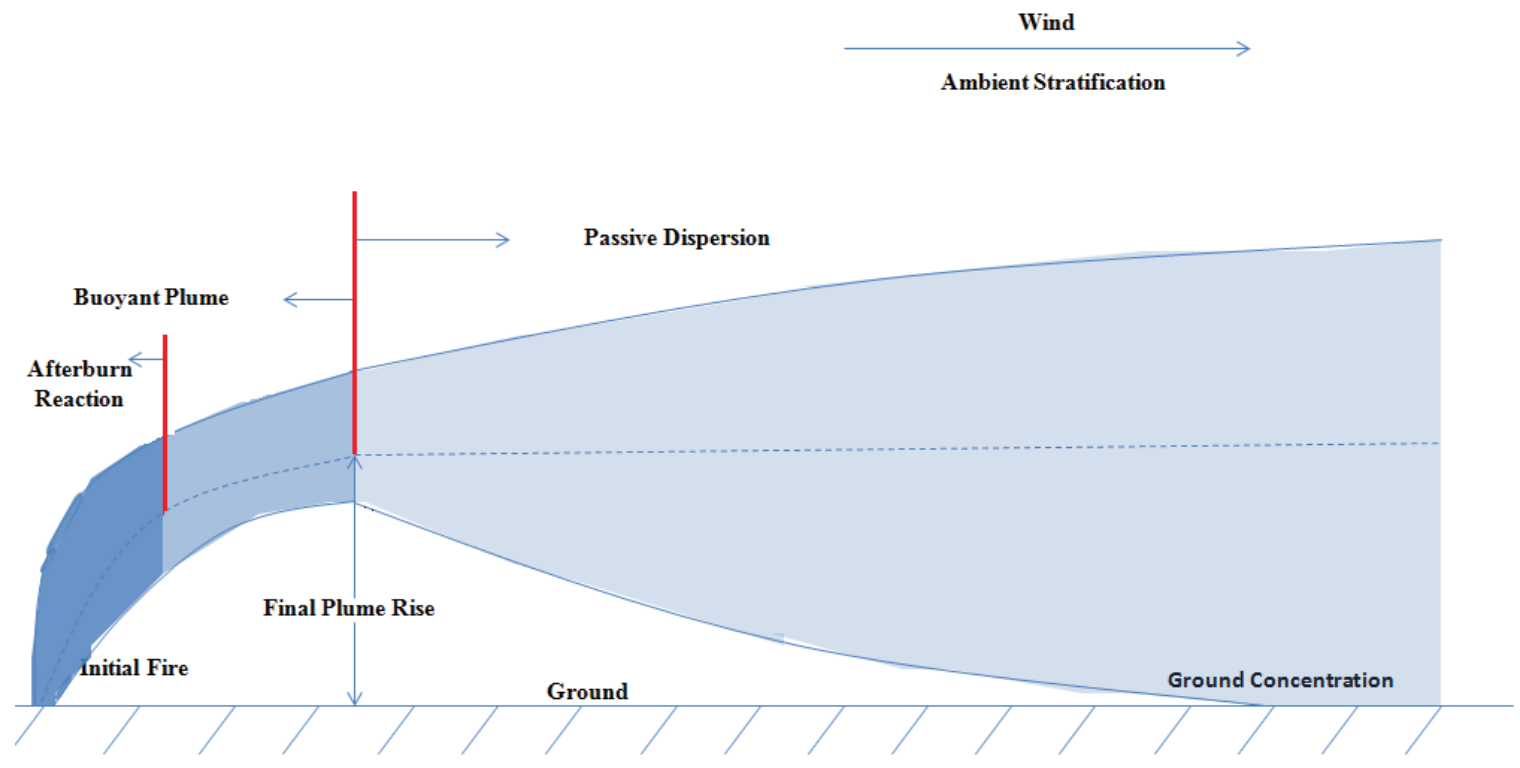

(b)

Fig. 1. Schematic of key processes considered in the ADORA model. (a) Puff from an explosion and (b) Plume from a fire.

\section{VALIDATION OF THE SPECIES PREDICTIONS}

Validation of all the modules of ADORA with a single set of test data is not practical because various test groups focused on different aspects of the overall solution. For example, some groups focused on measuring the cloud rise and buoyancy, while some focused on chemical composition and others focused on toxic dispersion. Accordingly, we have over the years validated various modules of ADORA using available test data from the literature. We present here the validation of the chemistry module. In clouds formed from fires and explosions, most of the high temperature reactions proceed in a similar way. Since reliable open field test data are available in the public domain only for explosive detonation tests, we focused on these for validation of our chemistry model. Several tests have been conducted by the US Department of Defense to generate validation data at various scales. These include the large scale open detonation tests at the Dugway Proving Grounds or DPG [16] ( 1000 kg charge per test), medium scale tests at the Nevada Test Site [17] (10s of kg per test) and small scale tests at Sandia National Laboratories' BangBox facility [18] ( $1 \mathrm{~kg}$ per test). Out of these only the large scale tests at DPG are true open detonation tests as the other two imposed constraints on cloud rise and amount of air available for afterburn reactions. In the DPG tests, the charge was ignited in the open and the cloud composition tracked versus time. Due to the difficulty associated with open air tracking of the cloud, these measurements focused only on the major species such as carbon dioxide $\left(\mathrm{CO}_{2}\right)$, carbon monoxide (CO), nitrogen oxide (NO), nitrogen dioxide $\left(\mathrm{NO}_{2}\right)$ methane $\left(\mathrm{CH}_{4}\right)$, Total Non-Methane Hydrocarbons (TNMH) and Volatile Organic Compounds (VOCs). The results from these tests were reported in the form of emission factors defined as the ratio of the mass of products in the cloud at the end of chemical reactions to the mass of original explosive material. The emission factors from four such tests in which RDX, TNT, Comp. B (a mixture of RDX and TNT) and Explosive D (ammonium picrate) are reported in Table 1.

Using the amounts of explosive charges detonated in each test and the environmental conditions (temperature, humidity, wind speed, elevation above mean sea level and atmospheric condition) as inputs, we calculated the cloud dynamics and composition with our model. ADORA predicts the time evolution and final yields of reaction products. Our predictions of emission factors are compared with field measurements for the final yields for $\mathrm{CO}_{2}, \mathrm{CO}, \mathrm{NO}, \mathrm{NO}_{2}, \mathrm{CH}_{4}$, Total Non Methane Hydrocarbons (TNMH) and benzene $\left(\mathrm{C}_{6} \mathrm{H}_{6}\right)$ produced in each test in Table 1. Here we used benzene as a surrogate for the VOCs. Twenty six out of 28 predictions are excellent while the remaining 2 predictions are off. Considering the complexity of the problem, this is a remarkable achievement. The fact that the program gives such a good 
agreement for so many species, without pre-ordaining the reaction, demonstrates ADORA's usefulness and uniqueness.

For comparison, Table 1 gives the predictions using conventional models such as ALOHA which do not have our reaction model. They assume that mixing with air and complete chemical reaction occurs instantaneously thereby producing a hot cloud that disperses as a buoyant gas [19]. These models require the user to specify the entrained air ratio (E/A) in the cloud as an input without any solid foundation. . E/A ratios had to be varied over a broad range (from 0.25 to 1.5 , with 1 being stoichiometric) to bracket the measured $\mathrm{CO}_{2}$ concentrations with predictions. Despite this, ALOHA failed to predict most species of interest and in the few cases where it does, the results are off significantly.

It is noteworthy that ALOHA uses semi-empirical plume rise formulae derived originally for continuous releases from power plant stacks where reaction is complete before release to the atmosphere. While being simple, such a treatment has additional shortcomings: (1) when complex reactions with several steps, components and phases are involved, the extent of reaction completion is not known a priori and intermediate species could be toxic and important; (2) the predicted reaction products and their amounts ignore the importance of the rate of ambient air entrainment and its effect on cloud temperature; (3) the dynamics of a buoyant cloud whose buoyancy is continuously fed by the heat released from chemical reaction is different from that where all the heat is released at once; and (4) the receptor of interest may be so close to the release location that the reacting cloud passes by the receptor before reaction completion. The net effect of such overly simplistic approaches is an underestimation of the effective release height, which leads to an overestimation of the toxic concentrations downstream.

Table 1. Comparison of emission factors predicted by ADORA and ALOHA (with pre-ordained entrained air ratios) with measurements from open detonation tests at the US Army Dugway Proving Grounds [16].

\begin{tabular}{|c|c|c|c|c|c|c|c|c|c|}
\hline Explosive & \multicolumn{2}{|c|}{ Data Source } & $\mathrm{CO}_{2}$ & $\mathrm{CO}$ & NO & $\mathrm{NO}_{2}$ & $\mathrm{CH}_{4}$ & TNMH & $\mathrm{C}_{6} \mathrm{H}_{6}$ \\
\hline \multirow{4}{*}{ RDX } & \multicolumn{2}{|c|}{ Field Measurement } & 570 & 31 & 0.9 & 0.6 & 1.2 & 1.3 & 0.069 \\
\hline & \multicolumn{2}{|c|}{ ADORA } & 590 & 13 & 0.91 & 0.7 & 1.7 & 2.2 & 0.076 \\
\hline & \multirow{2}{*}{ ALOHA } & $E / A=1.5$ & 520 & 46 & 0 & 0 & 0.01 & 0 & 0 \\
\hline & & $E / A=1.0$ & 590 & 3.2 & 0 & 0 & 0 & 0 & 0 \\
\hline \multirow{4}{*}{ TNT } & \multicolumn{2}{|c|}{ Field Measurement } & 1280 & 49 & 1.4 & 1.4 & 1.5 & 2.1 & 0.1 \\
\hline & \multicolumn{2}{|c|}{ ADORA } & 1260 & 58 & 2 & 1.2 & 1 & 0.85 & 0.14 \\
\hline & \multirow{2}{*}{ ALOHA } & $E / A=0.43$ & 920 & 280 & 0 & 0 & 0.19 & 0 & 0 \\
\hline & & $\mathrm{E} / \mathrm{A}=0.25$ & 1360 & 0 & 0.03 & 0 & 0 & 0 & 0 \\
\hline \multirow{4}{*}{ Comp. B ${ }^{\mathrm{a}}$} & \multicolumn{2}{|c|}{ Field Measurement } & 870 & 31 & 0.8 & 1 & 0.6 & 1.2 & 0.062 \\
\hline & \multicolumn{2}{|c|}{ ADORA } & 980 & 48 & 1.7 & 1 & 1.7 & 2.3 & 0.18 \\
\hline & \multirow{2}{*}{ ALOHA } & $E / A=0.67$ & 750 & 97 & 0 & 0 & 0 & 0 & 0 \\
\hline & & $E / A=0.43$ & 900 & 0 & 0.01 & 0 & 0 & 0 & 0 \\
\hline \multirow{2}{*}{ Expl. $D^{b}$} & \multicolumn{2}{|c|}{ Field Measurement } & 990 & 53 & 0.9 & 1.1 & 2.4 & 2 & 0.11 \\
\hline & \multicolumn{2}{|c|}{ ADORA } & 840 & 140 & 0.9 & 0.8 & 2 & 2.1 & 0.13 \\
\hline
\end{tabular}

a Comp. B is an explosive containing approximately 59.5\% of RDX, 39.5\% of TNT and 1\% wax

${ }^{\mathrm{b}}$ Expl. D is ammonium picrate

\section{SAMPLE RESULTS AND DISCUSSION}

We present results from ADORA simulations for two types of scenarios. The first involves the detonation and afterburn reactions of an explosive material continuing the validation case. The second type focuses on fires involving reactive chemicals. 


\section{Detonation and Afterburn Reactions of an Explosive}

Here we present results from ADORA simulations for the detonation of $400 \mathrm{~kg}$ of Comp. B. We discussed the composition of the cloud at the end of chemical reactions (expressed in terms of emission factors) under the validation. The discussion below focuses on cloud evolution during the detonation, afterburn and buoyant cloud rise stages before passive dispersion sets in.

Explosives are designed to produce a large number of molecules to maximize the ability to produce pressure at the expense of reaction completion (or conversion to completely oxidized state). The hot products of detonation continue to burn as air is entrained into the resulting puff (a process referred to as afterburn). Calculations with ADORA show that the main products of afterburn reactions are $\mathrm{CO}_{2}$ and $\mathrm{H}_{2} \mathrm{O}$ and are non-toxic. Minor species include CO, nitrogen oxides (NOx) and VOCs which are toxic, but their concentrations and dosages are low as presented in Table 1. However, accounting for detonation and afterburn sequentially has a significant effect on the cloud temperature as more time is allowed for heat loss. The corresponding effect on cloud rise and downwind dispersion is also significant. For local meteorological conditions, we assumed a pressure of 1 atmosphere, a temperature of $20^{\circ} \mathrm{C}$, a humidity of $55 \%$, a wind speed of $3 \mathrm{~m} / \mathrm{s}$ and a neutral atmospheric stability parameter of D.

The cloud temperature is plotted as a function of time and distance in Fig. 2 (a) and (b). The cloud is extremely hot at the end of detonation reaction. The afterburn reactions between the products of the detonation and the entrained air occur very fast (less than 1 second). The rate of heat release due to chemical reactions during this time is less than the rate of heat loss (due to a combination of radiation and dilution) and the net effect is a drop in cloud temperature. Most of the chemical reactions end in about $1 \mathrm{~s}$. The cloud is still quite hot and buoyant at this time. It continues to rise up, disperse downwind, entrain ambient air and cool down. It takes about 55 seconds for the cloud to cool down to the ambient temperature. It travels about $180 \mathrm{~m}$ (590 feet) downwind during this time. The cloud radius increases to about $58 \mathrm{~m}$ (= 190 feet) and its height to $264 \mathrm{~m}$ (= 865 feet) at the transition to passive dispersion.

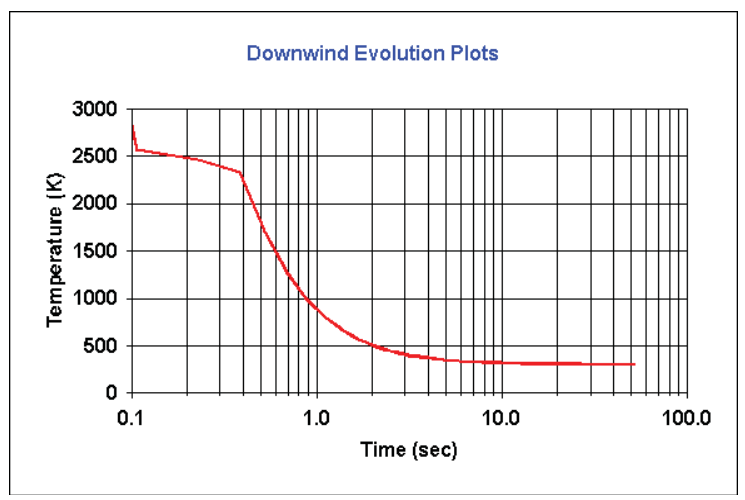

(a)

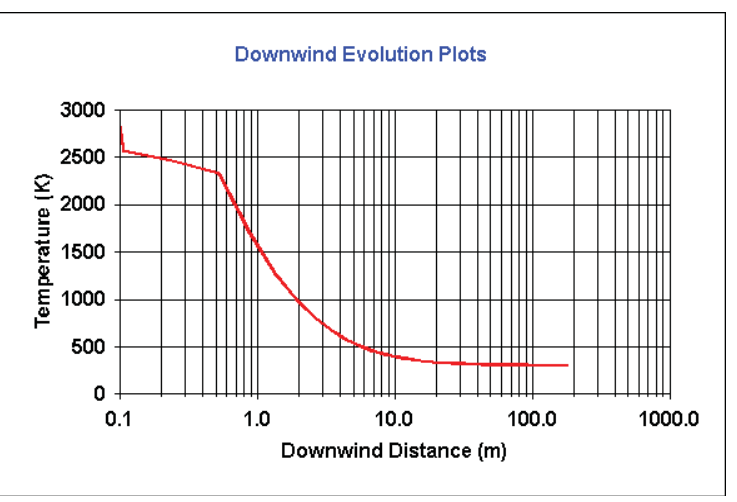

(b)

Fig. 2. Cloud temperature as a function of (a) time and (b) distance.

For completion, the blast and thermal effects produced by this detonation are shown in Fig. 3. The side-on overpressure from the blast generated by the detonation is plotted as a function of distance in Fig. 3 (a). The blast overpressure decreases rapidly as the distance from the detonation increases. We stopped the calculations at a distance when the transition to passive dispersion occurs, but the blast travels even further. The blast overpressure decreases below $6000 \mathrm{~Pa}$ where it does not cause any significant structural damage $200 \mathrm{~m}$ away from the detonation location. Using the cloud temperature, we calculated the maximum emitted radiation heat flux from the cloud versus downwind distance and plotted it in Fig. 3 (b). The maximum emitted radiation heat flux for this case decreases below pain threshold levels when the cloud travels about $20 \mathrm{~m}$ from the detonation location. 


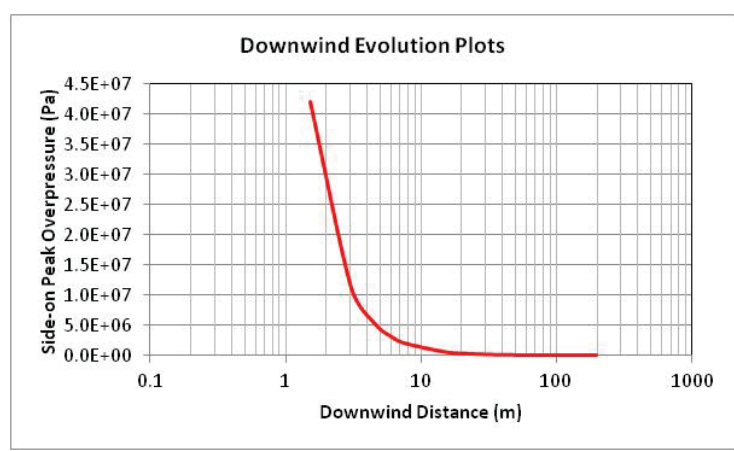

(a)

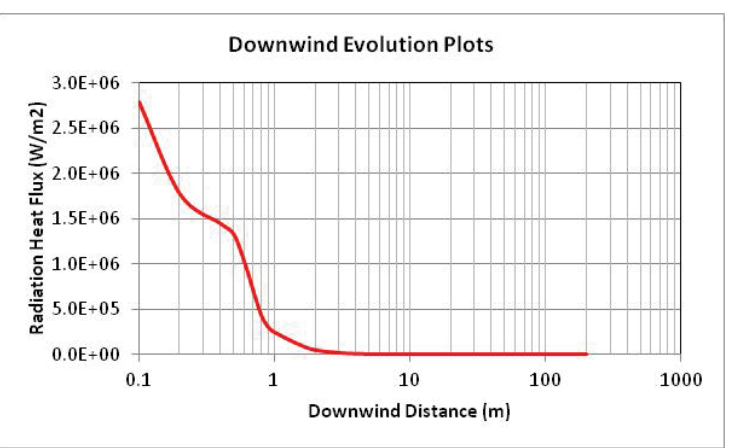

(b)

Fig. 3. Blast overpressure and maximum emitted heat flux from the cloud versus distance.

\section{Chemical Fires}

To illustrate the breadth of chemistries that can be handled by ADORA two examples of chemical fires are given below. The first is an industrial fire involving a pyrotechnic plant while the second is a chemical warehouse fire. Since the early chemistry is rather insensitive to the normal range of meteorological parameters, typical values have been used in the calculations below, namely, 1 atmosphere, 20 C, 55\% humidity, $8 \mathrm{~m} / \mathrm{s}$ wind speed and a neutral atmospheric stability parameter of D.

\section{Example 1: Industrial Fire}

The first example is of an industrial fire involving $35 \mathrm{~kg}$ Diesel fuel, $33 \mathrm{~kg}$ Magnesium (Mg), $19 \mathrm{~kg}$ Teflon $\left(\left(\mathrm{C}_{2} \mathrm{~F}_{4}\right)_{\mathrm{n}}\right.$ with a fluorine content of $61 \%$ ), and $3 \mathrm{~kg}$ Viton $\mathrm{A}$ (with a fluorine content between $66 \%$ and $70 \%$ ). The assumption of thermochemical equilibrium produces a pool of products which evolves in time as the temperature of the cloud changes. Then, these products are selectively frozen as the temperature drops due to cloud dilution by air. For example, an examination of kinetic parameters suggests that the reaction rate for the decomposition of HF becomes very slow when the temperature decreases below $1400 \mathrm{~K}$. Accordingly, we assume that the HF consumption stops when the cloud temperature decreases below 1400 K. Note that this does not prevent the formation of additional HF with time. Using a similar approach, we have developed a database for freezing temperatures of key species. Accordingly, we freeze various products such as $\mathrm{CO}, \mathrm{NO}_{2}$, $\mathrm{HF}$, and $\mathrm{MgO}$ at various temperatures between $2350 \mathrm{~K}$ and $1300 \mathrm{~K}$ as the cloud cools down. Implementation of these freezing temperatures in our model yields a much faster calculation than incorporation of the chemical reaction rates.

Fig. 4 presents the evolution of temperature and concentrations of key product species as a function of time. Note that the temperature starts high and unlike the above detonation case it increases further as air is entrained into the cloud, and past $0.7 \mathrm{~s}$ it decreases due to dilution. The CO concentration increases during the first $0.5 \mathrm{~s}$ due to chemical reactions and then it decreases due to dilution. NO begins to form after half a second while the formation of $\mathrm{NO}_{2}$ is negligible. Tradeoffs between different fluorine and magnesium containing species, such as $\mathrm{Mg}(\mathrm{g}), \mathrm{MgF}$ radical, and various phases of $\mathrm{MgF}_{2}$, are predicted by the code as they appear and disappear. Immediately noticeable is the fact that one of the usual choices for end products in any other model, $\operatorname{MgF}_{2}(\mathrm{~s})$, is not formed until rather late in cloud evolution. Also, the split between the final $\mathrm{MgO}$ and $\mathrm{MgF}_{2}$ (s) is predicted as both oxidizers compete for the $\mathrm{Mg}$. Clearly, $\mathrm{MgO}$ is the predominant final product partly due to the abundance of oxygen entrained in the form of ambient air and due to the formation of HF (and freezing done in our model). If the freezing assumption is not invoked and conventional thermochemical equilibrium is assumed at all temperature and times, the model will yield $\mathrm{MgF}_{2}$ as the main reaction product. If one had ignored this key step assuming arbitrarily that all the $\mathrm{Mg}$ converts to $\mathrm{MgF}_{2}(\mathrm{~s})$, the resulting heat release, cloud fireball temperature, entrainment rate and ultimately rise height would be significantly off. Instead, the code gives a more realistic balance of the produced species from which to calculate the all-important toxic HF concentration as well as puff dynamics. During these transformations, the HF concentration increases by 3 orders of magnitude. 
Most of the chemical reactions end during the first one second. Later, the concentration variation is dominated by the entrainment of ambient air into the cloud, i.e., atmospheric dispersion. Such dispersion can be calculated by ADORA or alternatively, the code can calculate (1) the final emission factors for the various species and the cloud temperature, size and rise height (at about $1 \mathrm{~s}$ ) and pass the information to another buoyant dispersion code; or (2) continue the calculation until the cloud buoyancy is dissipated (at about 5 s) and pass the final cloud properties to a passive dispersion code.

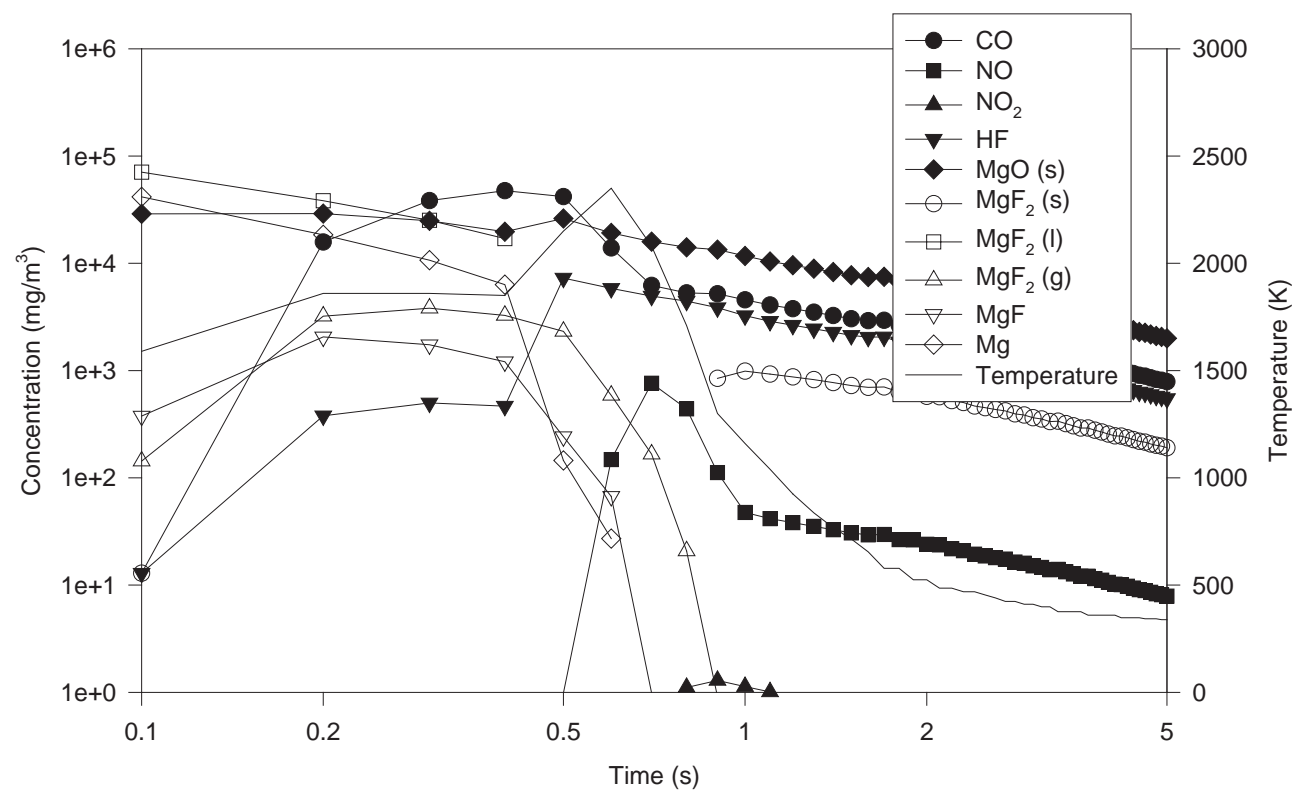

Fig. 4. Time dependent temperature and chemical composition of the cloud from a fire involving magnesium, Teflon, Viton and diesel.

For the above conditions, the cloud reaches an equilibrium height of $160 \mathrm{~m}$ at downwind distance of $360 \mathrm{~m}$ in about 50 seconds after the release. The concentration contours of HF are plotted as a function of downwind distance in Fig. 4. The dangerous concentration are very near the source but rise with distance as indicated in the cloud centerline concentration in an x-z plane in Fig. 5 (a). Away from the centerline and from the height where the cloud has risen, the concentrations are negligible as shown in Fig. 5 (b). Unfortunately, measured data are not available for direct comparison with the above predictions.

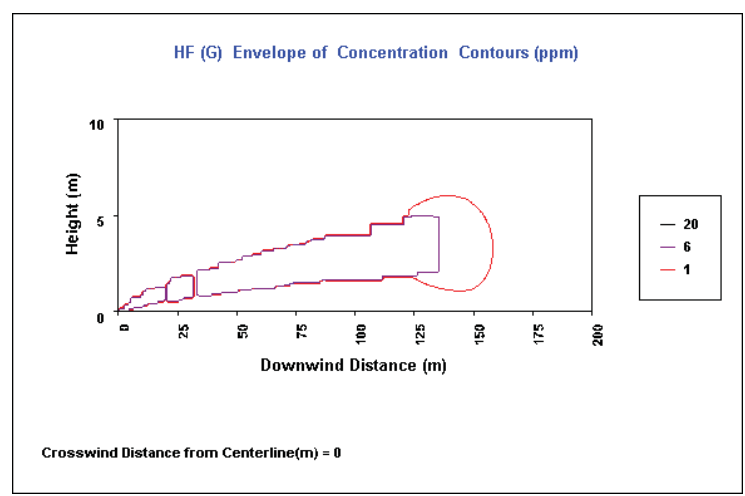

(a) $x-z$ plane

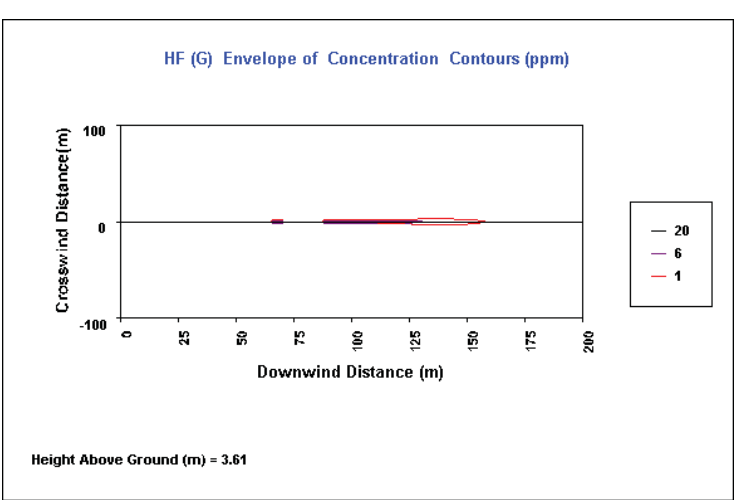

(b) $x-y$ plane

Fig. 5. Envelope of HF concentration contours from a fire involving magnesium, Teflon, Viton and diesel. 


\section{Example 2: Warehouse Fire}

The composition of the burning materials in the second fire was: $24 \mathrm{~kg}$ red phosphorus (P), $15 \mathrm{~kg}$ manganese dioxide $\left(\mathrm{MnO}_{2}\right)$, $3 \mathrm{~kg}$ magnesium (Mg), $1.4 \mathrm{~kg}$ zinc oxide ( $\left.\mathrm{ZnO}\right), 1.5 \mathrm{~kg}$ linseed oil and $45 \mathrm{~kg}$ of water. Fig. 6 shows temperature and chemistry evolution for this fire similar to the discussion of Fig. 4. The initial temperature of the fire is about $1600 \mathrm{~K}$. It increases with time during the first $0.3 \mathrm{~s}$ to about 1900 $\mathrm{K}$ mainly due to the reaction of phosphorus and magnesium with oxygen. The relatively low peak temperature is due to the presence of large amounts of materials with low reactivity such as $\mathrm{MnO}_{2}$ and $\mathrm{ZnO}$. Entrainment of ambient air and heat losses from the cloud cause the temperature to drop rapidly at later times. Using the constrained equilibrium approach, ADORA calculated the concentrations of CO, NO, $\mathrm{NO}_{2}, \mathrm{P}_{4} \mathrm{O}_{10}, \mathrm{H}_{3} \mathrm{PO}_{4}, \mathrm{Mg}_{3}\left(\mathrm{PO}_{4}\right)_{2}, \mathrm{Zn}_{3}\left(\mathrm{PO}_{4}\right)_{2}, \mathrm{ZnO}$ and $\mathrm{MnO}$ - which are 'frozen' progressively as the cloud temperature drops below their corresponding freezing temperatures from our database. Relative to the previous example, more exotic (but less toxic) chemicals are major products of this release, including phosphates, phosphoric acid, metal oxides, and various phosphorus oxides. The code predicts the correct $\mathrm{P}_{4} \mathrm{O}_{10} / \mathrm{H}_{3} \mathrm{PO}_{4}$ ratio typical of a phosphorus burn (near 5\%). We can also see the same kinds of tradeoffs that if ignored, would lead to inappropriate minor product species emission factors and cloud temperature and rise characteristics.

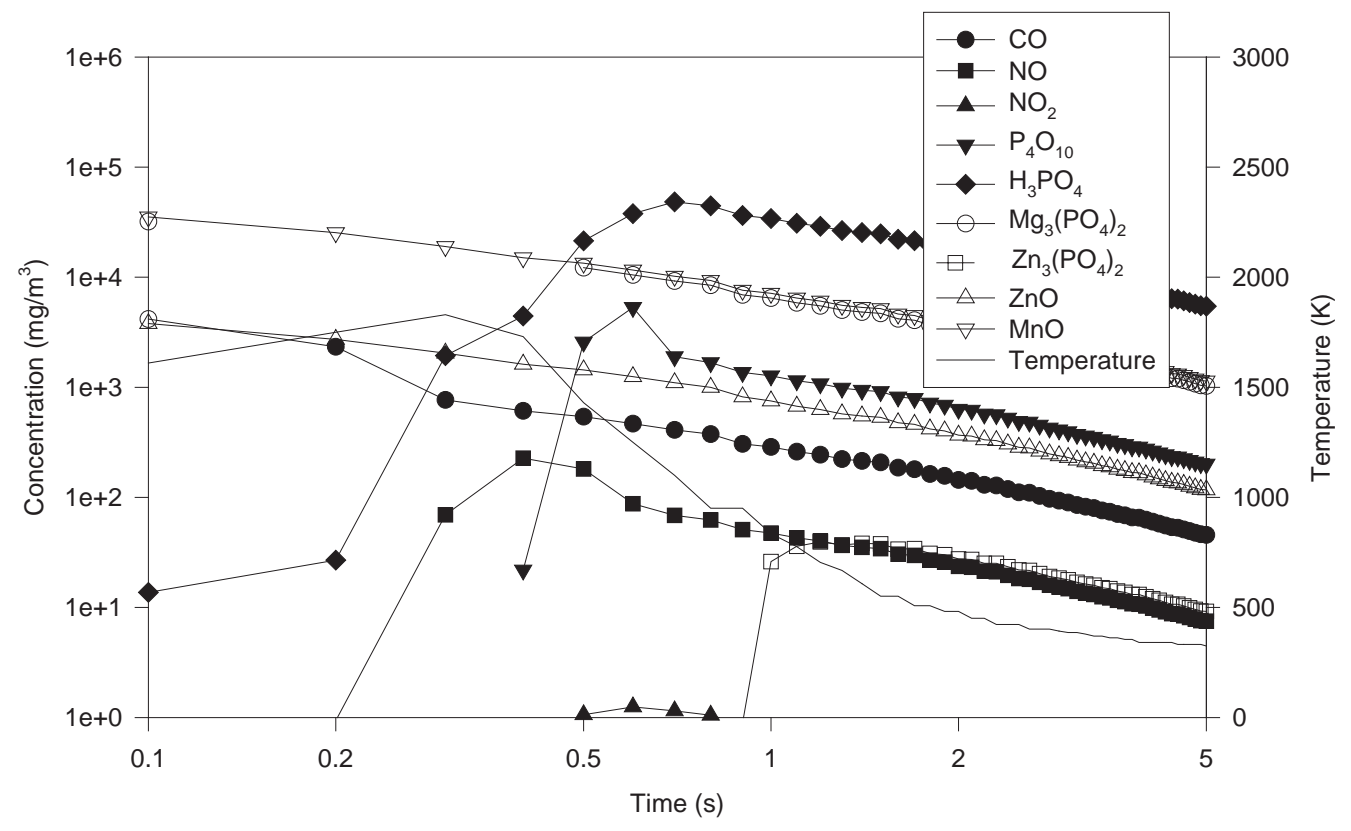

Fig. 6. Time dependent temperature and chemical composition of the cloud from a fire involving manganese dioxide, red phosphorus, linseen oil, zinc oxide, magnesium and water.

\section{CLOSURE}

The ADORA model presented here predicts the toxic emissions and cloud evolution from chemical fires and explosions accounting for variations in temperature, chemical composition, cloud size and locations with time and space. The model uses a computationally efficient approach called "constrained equilibrium" which is essentially an approximate way of incorporating reaction kinetics into equilibrium analysis. This approach combines the speed of classical equilibrium based approaches with the accuracy of kinetics based approaches. The reaction chemistry is coupled with cloud dynamics to generate the downwind toxic contours sought after in hazard calculations. The model architecture is designed to be flexible, and its general algorithms allow easy adaptation to a wide range of applications. For example, they can also pass the predicted emission factors and cloud characteristics at the end of combustion or at the end of cloud rise 
to classical dispersion models. As such ADORA can bridge the gap between source specification and meteorological dispersion by careful consideration of near-source effects including toxic product formation, heat release and cloud dynamics.

ADORA predictions agree well with data from four large scale detonation tests performed at the Dugway Proving Grounds [16] and this serves as validation for the chemistry module. In addition to the cloud composition at the end of chemical reactions (that was measured in the tests), ADORA predicts the cloud dimensions, location and temperature versus time and space, all needed for dispersion hazard analysis. Similarly, for two example chemical fires involving metals, fluorinated polymers and hydrocarbon fuels, ADORA predicts the cloud temperature and composition versus time and space. In addition to the final stable products, it predicts the formation and consumption of reaction intermediates.

ADORA generates a wealth of data that has been used in hazard analysis of chemical plants and chemical warehouse fires. It has also been used in preparing environmental permit applications, designing large scale tests, investigating accidents, planning the emergency response, and devising and evaluating methods to protect against accidents involving toxic emission from reactive chemicals.

\section{REFERENCES}

[1] Petersen, K. E., and Markert, F., (1991) Assessment of fires in chemical warehouses. An overview of the TOXFIRE project, Risø National Laboratory, Roskilde, Denmark Risø-R-932(EN).

[2] McGratten, K.B., Putori, A.D., Twilley, W.H., and Evans, D.D. (1993) Smoke Plume Trajectory of In Situ Burning of Crude Oil in Alaska, Technical Report NISTIP 5273, NIST, Gaithesburg, Maryland.

[3] Walton, W.D., "Smoke measurements using a helicopter transported sampling package," Proceedings of the $17^{\text {th }}$ Arctic and Marine Oil Spill Program (AMOP) Technical Seminar, pages 735-764. Environment Canada, Emergencies Science Division, Ottawa, Ontario, Canada, 1994.

[4] Fingas, M.F., “The Newfoundland Offshore Burn Experiment- NOBE,” Proceedings of the 1995 International Oil Spill Conference, pages 123-132, Publication 4620, American Petroleum Institute, 1995.

[5] Baum, H.R., McGratten, K.B., and Rehm, R.G., "Numerical simulation of smoke plumes from large oil fires,” Atmospheric Environment, 30 (24): 4125-4136, 1996.

[6] Laursen, K.K., "Emission factors for particles, elemental carbon, and trace gases from the Kuwaiti oil fires”, Journal of Geophysical research, Vol. 97, No. D13, pp. 14,491-14,497, 1992.

[7] Argyropoulos, C.D., Sideris, G.M., Christolis, M.N., Nivolianitou, Z., and Markatos, N.C., "Modelling pollutants dispersion and plume rise from large hydrocarbon tank fires in neutrally stratified atmosphere," Atmospheric Environment 44, no. 6: 803-813, 2010.

[8] Markatos, N. C., Christolis, C., and Argyropoulos, C.D., "Mathematical modeling of toxic pollutants dispersion from large tank fires and assessment of acute effects for fire fighters," International journal of heat and mass transfer 52.17: 4021-4030, 2009.

[9] Mohan, R., Walton, H. A., Thomson, D., Webster, H., Wilkinson, P., Grundy, C., Murray, V., and Leonardi, G., "The Buncefield Oil Depot Fire of 2005: Potential Air-Pollution Health Impacts Under Alternative Meteorological Scenarios," PLoS currents 4, 2012.

[10] Altarawneh, M. and Dlugogorski, B.Z., "Mechanisms of Pollutant Formation in Fires,” Proc. Of the $7^{\text {th }}$ International Seminar on Fire and Explosion Hazards, pp. 27-36, 2013.

[11] Tuovinen, H., Blomqvist, P., and Saric, F., "Modelling of hydrogen cyanide formation in room fires," Fire Safety Journal 39.8: 737-755, 2004.

[12] Blomqvist, P., Emissions from Fires: Consequences for Human Safety and the Environment, PhD Thesis, Department of Fire Safety Engineering, Lund Institute of Technology, Lund University, Lund 2005. 
[13] http://www.csb.gov/on-first-anniversary-of-investigation-board-releases-data-on-167-seriousreactive-incidents/

[14] Moussa N. A., Zhang X.J., Groszmann D.E., Beach A.B., and Noland R.B. "Reacting Puff Rise,” JANNAF Safety and Environmental Protection Subcommittee Joint Meeting, Tampa, Florida, 1995.

[15] Moussa, N. A., Masonjones, M.C. and Zhang, X.J, "Accurate Prediction of Emission Factors by ADORA," Presented at the 1998 Global Demilitarization Symposium and Exhibition in Coeur D’Alene, Idaho, May 11-14, 1998.

[16] Johnson, M., (1992) Development of Methodology and Technology for Identifying and Quantifying Emission Products from Open Burning and Open Detonation Thermal Treatment Methods, Volume 2, Part A, Report No. ADA250736, Headquarters U.S. Army Armament, Munitions and Chemical Command.

[17] Watkins, B. E. et al., "Phase III Demonstrations Detonations of Conventional Weapons: $155 \mathrm{~mm}$ High Explosive M107 Projectiles", Prepared for the Defense Ammunition Center under the DoD/DoE Joint Demilitarization Technology Program, 2000.

[18] Mitchell, W.J. and Suggs, J.C., (1998) Emission factors for the disposal of energetic materials by open burning and open detonation (OB/OD), EPA/600/R-98/103.

[19] ALOHA the atmospheric dispersion model in the CAMEO software suite developed by EPA for emergency response planning. http://www.epa.gov/osweroe1/content/cameo/what.htm 